Original published version available at:

http://dx.doi.org/10.1504/IJSS.2004.005700

B. Heidkamp, A Pohl, U Schiek, F.Klinkenberg, J. Hynynen, A Sieber, P. Christ, T. Owens, J. Cosmas, T. Itagaki and F. Sun, 2004, “Demonstrating the feasibility of standardized application program interfaces that will allow mobile/portable terminals to receive services combining UMTS and DVB-T", International Journal of Service and Standards, Vol.1, No.2, pp.228-242

\title{
Demonstrating the feasibility of standardized application program interfaces that will allow mobile/portable terminals to receive services combining UMTS and DVB-T
}

Bettina Heidkamp

Rundfunk Berlin-Brandenburg, Marlene-Dietrich-Allee 20, 14482 Potsdam, Germany

Alexandra Pohl,

Rundfunk Berlin-Brandenburg, Marlene-Dietrich-Allee 20, 14482 Potsdam, Germany

\section{Ulrich Schiek}

Institute for Communications Technology, Braunschweig, Technical University, Pockelsstrasse 14, 38106 Braunschweig, Germany

\section{Frank Klinkenberg}

Institute for Communications Technology, Braunschweig, Technical University, Pockelsstrasse 14, 38106 Braunschweig, Germany

\section{Juha Hynynen}

Netikos Finland Oy, Kluuvikatu 4B, 00100, Helsinki, Finland

Andreas Sieber

Institut fuer Rundfunktechnik, Gmbh, Floriansmuehlstrasse 60, 80939, Muenchen, Germany

Peter Christ 


\title{
Thomas Owens
}

Department of Electronic and Computer Engineering, Brunel

University, Uxbridge, Middlesex, UB8 3PH, United Kingdom

\section{John Cosmas}

Department of Electronic and Computer Engineering, Brunel University, Uxbridge, Middlesex, UB8 3PH, United Kingdom

\section{Takebumi Itagaki}

Department of Electronic and Computer Engineering, Brunel University, Uxbridge, Middlesex, UB8 3PH, United Kingdom

\section{Frank Sun}

Department of Electronic and Computer Engineering, Brunel University, Uxbridge, Middlesex, UB8 3PH, United Kingdom

\begin{abstract}
Crucial to the commercial exploitation of any service combining UMTS and DVB-T is the availability of standardized API's adapted to the hybrid UMTS and DVB-T network and to the technical limitations of mobile/portable terminals. This paper describes work carried out in the European Commission Framework Program 5 (FP5) project CONFLUENT to demonstrate the feasibility of such Application Program Interfaces (API's) by enabling the reception of a Multimedia Home Platform (MHP) based application transmitted over DVB-T on five different terminals with parts of the service running on a mobile phone.
\end{abstract}

Keywords: Services, Standards, Combining UMTS and DVB-T, MHP

Reference to this paper should be made as follows: Heidkamp, B., Pohl, A., Schiek, U., Klinkenberg, F., Hynynen, J., Sieber, A., Christ, P., Owens, T., Cosmas, J., Itagaki, T. and Sun, F. (2004) 'Demonstrating the feasibility of standardized application program interfaces that will allow mobile/portable terminals to receive services combining UMTS and DVB-T', Int. J. Services and Standards, Vol. 1

Biographical notes: Bettina Heidkamp is employed by RBB to coordinate multimedia research and development for RBBs Department Production \& Operation. Since 2001 Alexandra Pohl has been working for RBB's Innovation Projects. She is currently managing RBB's project activities for the FP6 Integrated Project ENTHRONE (IST-507637). Juha Hynynen was Managing Director of NETikos Finland Oy. Andreas Sieber is a Research Engineer with the Institut fuer Rundfunktechnik. Peter Christ is Head of Section, International Transfer, at T-Systems Nova. Ulrich Schiek and Frank Klinkenberg are Research Assistants with the Institute for Communications Technology, Braunschweig Technical University. Thomas J. Owens is a lecturer, John Cosmas is a Professor, Takebumi Itagaki is a 
lecturer, and Frank Sun a Research Assistant all with the Department of

Electronic and Computer Engineering, Brunel University.

\section{$1 \quad$ Introduction}

The current modus operandi of wireless transmission systems is each individual system operates on its own. This means that UMTS/GPRS provides communication services at much less than 2Mbit/s whilst DVB-T provides broadcast services of about $15 \mathrm{Mbit} / \mathrm{s}$ (the current DVB-T mode in Germany and UK). By combining UMTS/GPRS and DVB services the broadband of DVB-T is combined with the interactive medium of UMTS/GPRS to create a mobile terminal that can be used to provide significant services. A UMTS network with an integrated DVB-T downlink could be enhanced with a direct link between the broadcaster and an Internet Service Provider. Portable/handheld consumer terminals for hybrid services coupling broadcast and broadband services would thus provide widely available alternative platforms for interactive TV-based services as well as for Internet based services. Crucial to the commercial exploitation of any service combining UMTS and DVB-T is the availability of standardized API's adapted to the hybrid network and to the technical limitations of mobile/portable terminals. The work of the European Commission FP5 project CONFLUENT included the provision of content to create and deliver an application for an exemplary service scenario and the software API's necessary to demonstrate that the application could be received on a variety of terminals. At the end of the CONFLUENT project in September 2003 software API's enabling the reception of a significant MHP-based application developed in the project on five different terminals of differing capabilities with parts of the service running on a mobile phone had been demonstrated. This paper describes the demonstration set-up, the application, the terminals and the API's.

\section{The set-up of the demonstration}

The demonstration was delivered at IFA 2003 (International Radio and TV Exhibition) in Berlin. The demonstration showed the "Local Guide Potsdam" - a module of an MHP-based Travel Guide application, developed by Institute for Communications Technology, Braunschweig, Technical University (TU-BS) in cooperation with Rundfunk Berlin-Brandenburg (RBB), running on five different terminals. In addition, a solution was demonstrated of mobile users transferring Local Guide Potsdam content from one of the terminals, an automotive mobile platform, to a commercially available mobile phone. The Local Guide Potsdam was shown on the following five terminals:

1 A static main MHP-automotive terminal with a VW Touareg dashboard (at the Deutsche Telekom Booth at IFA 2003) referred to as the CONFLUENT IFA showcase. This showcase presented mobile television and other MHP-applications transmitted via DVB-T that included the Local Guide Potsdam, as well as a Theatre Guide developed by TU-BS and the standard applications provided by the public broadcasters and T-Systems. To enable attractive automotive services TUBS developed a navigation system and a new application launcher/administrator 
called Navigator providing access to the MHP-based applications. This demonstration was based on a standard PC running the MHP Reference Implementation from IRT. In addition to the standard MHP interfaces, TU-BS offered a number of software extensions for the mobile environment such as specific API's to provide access to the navigation systems, to support Bluetooth communications and a Bluetooth interface that enabled the user to transfer Local Guide content to a mobile phone.

2 Activy set-top box (at the Deutsche Telekom Booth at IFA 2003).

3 A mobile automotive terminal equipped with the MCP automotive platform. Here, an embedded MHP implementation was integrated by ICT Embedded B.V. on a Philips Nexperia board. This demonstration showed the first embedded MHP implementation prototype for the car. (A mobile demonstration outside the IFA 2003 exhibition area supported by Audi and ICT Embedded B.V.).

4 A terminal with a MHP Reference Implementation SW stack. (at the IRT Booth at IFA 2003).

5 A mobile terminal in a car provided by IRT (a mobile demonstration outside the IFA 2003 exhibition area). Apart from running the Travel Guide Application, here, IRT showed the performance of MHP based services at high speed.

T-Systems was granted a licence to broadcast on channel 51 in Berlin during IFA 2003. Three DVB-T transmitters located at Alexanderplatz, Schäferberg, and Scholzplatz (3-5kW) were used. The broadcasting parameters were as follows:

1 Broadcast Mode: 16 QAM, 8 k, 2/3

2 Protection Ratio GI = 1/8

3 The maximum data rate of the channel: $14.75 \mathrm{Mb} / \mathrm{s}, 300 \mathrm{~kb} / \mathrm{s}$

Within the multiplex three TV programmes and a large number of datacast services (MHP, datacast, etc.) were broadcast.

T-Systems used the Alticast Play-out system. The CONFLUENT project application was broadcast within an object carrousel delivering about 300kbit/s. This was sufficient to make text and image content available every minute. The total amount of Travel Guide data was about 650 kBytes. The major part (about 450 kBytes) is resources. So it took about 17 to 18 seconds to transmit the whole application - including the programme code, the pictures, the texts and additional meta information e.g. for speech control. Of course the exact time depends on the "entering point" in the carousel. The transmission of MPEG4 videos took several minutes due to the volume of data. This issue is not that critical because after starting the application it takes the user some time to navigate through the menus and come to the first video offer. This time can be used to receive the videos in the background. 
For the underlying service scenario, The Metropolitan Mobility Guide, the target group are commuters in a large conurbation area. The multimedia service information is to be available inside and outside the car environment. The usage context is mobile (car) plus mobile (phone) and the content focus is a modular information service: news, entertainment, events, Local Guide (guided tours and sightseeing), navigation.

For the CONFLUENT project the Local Guide part of the Metropolitan Mobility Guide was taken as the content example for the Travel Guide Application. This takes the form of the "The Local Guide Potsdam", see Figure 1.

Figure 1 Sample screen from the Local Guide Potsdam

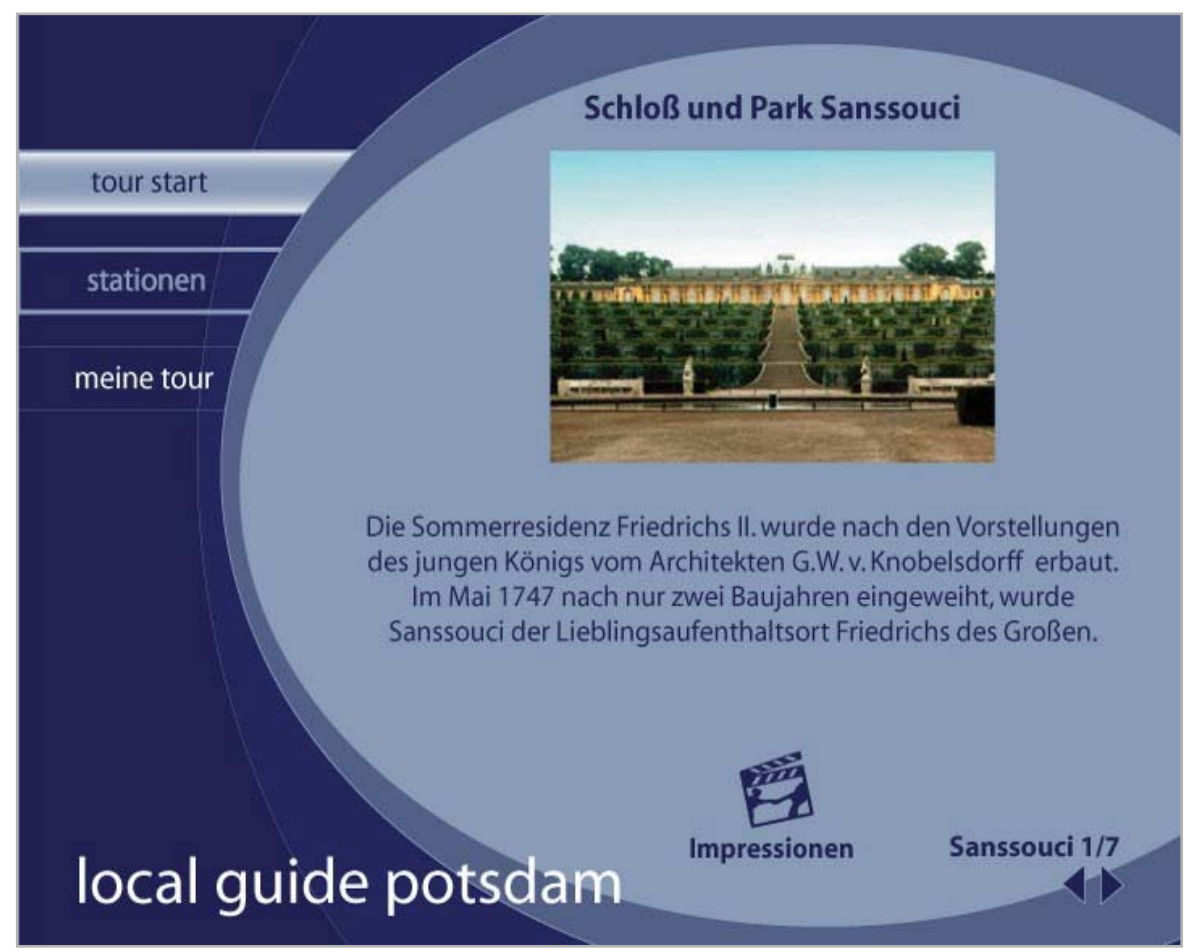

The Local Guide Potsdam is a mobile multimedia application developed for delivery over DVB-T to be received in static or mobile (automotive and pedestrian) usage situations. It leads to the most attractive sights of Unesco World Heritage Town Potsdam and offers a combination of text, pictures and MPEG4-videoclips. Content modules of the local guide can be transferred to a mobile phone via Bluetooth. The content was selected from RBB's content pool deriving from different content sources such as TV programmes and Websites.

The use case of the Local Guide is a mobile user (e.g. tourist) entering a specific town. On their mobile terminal (car or handheld) the user receives the alert that an application is available in the broadcast channel. Starting the application, the user gets information related to this specific town e.g. description of sights, events, and history. The user can browse through the content that consists of texts, pictures and short MPEG4 video clips. All actions required to control the application can be initiated either via classical car input devices (button, touch screen) or via voice. Due to the fact that the application can also get access to the navigation system of the terminal, location based support is available at any time, see Figure 2. So the user can ask for a map to display the position of all the sights described in the Local Guide. The user 
can also start a guided tour through the town. When the user arrives at a destination of

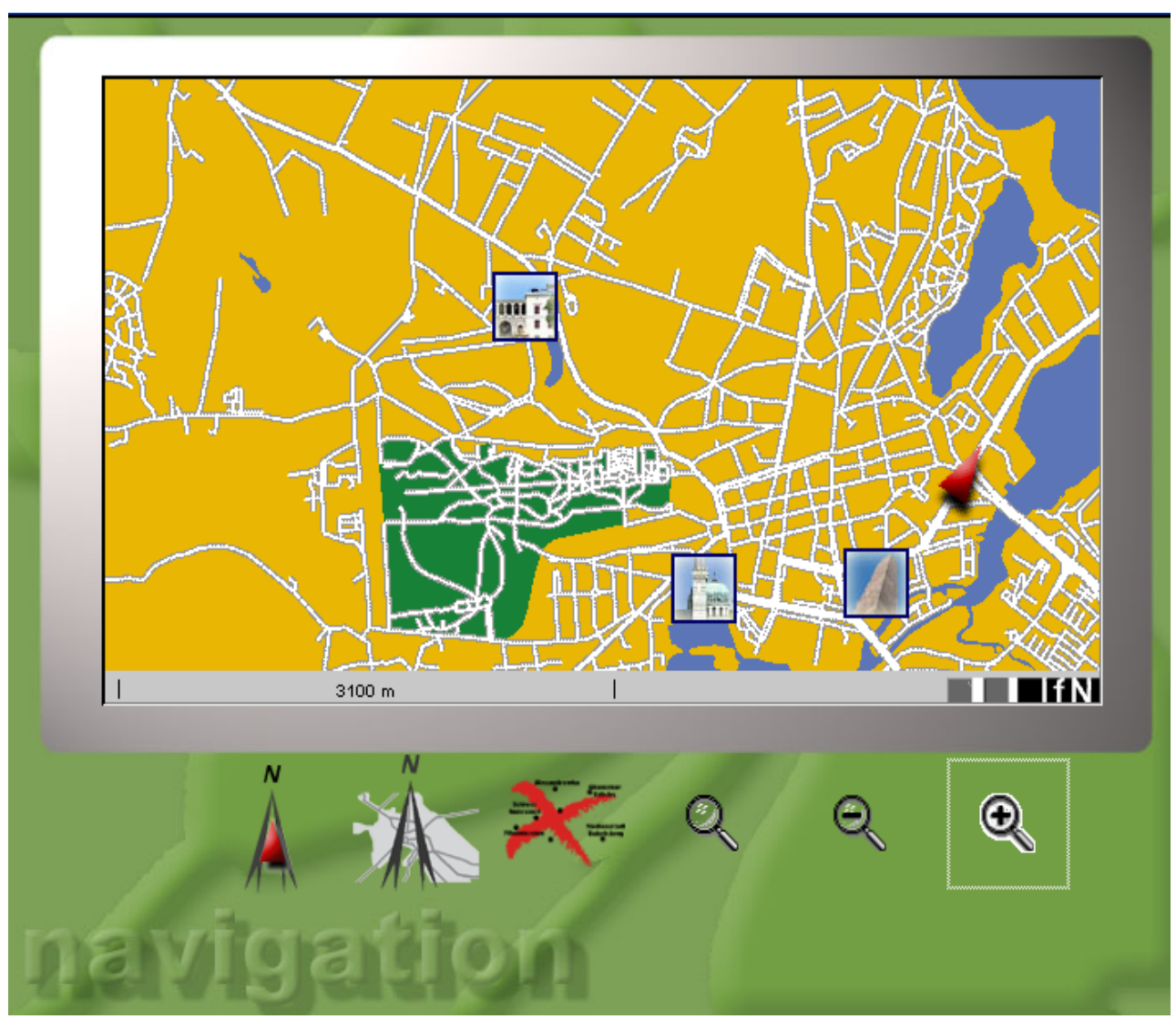

that

tour

the

Travel Guide presents the user with the related information. If the user wants to park/leave their car then it is possible to transfer the content, which they already received with a high bit rate via broadcast for free, via the short range communication standard Bluetooth to a commercially available mobile phone. A small Symbianbased application starts to parse and scale the content for an adapted presentation on the phone.

Figure 2

Sample screen from the Navigation Application 
In addition to users such as car drivers more specific possible target groups for such a service could be both local and external tourists. The basic idea is that the Local Guide provides them with all kinds of information they need in order to find their way around in one specific area, combining "inside and outside car/pedestrian usage”. Once activated, information can not only be received over DVB-T in the car while driving around but also, getting out of the car, the content modules can be transferred to a mobile phone to be taken away. The Local Guide Potsdam offers five different usage options:

1 Information on demand: Users can receive all available information on each sight individually and on demand according to their personal interests - either stationary or while moving.

2 Guided Tour: Users can choose a "Guided Tour" and will then be navigated along a certain itinerary from one sight to another.

3 Individual Tour: Users can also compose their personal guided tour, choosing sights and their specific order individually.

4 Automatic Navigation: Users can choose one specific sight to be "navigated" to via the navigation application.

5 Location Based Service: While driving around (either to individual sights or while being guided along a certain tour), information on the respective sights is provided automatically via GPS.

From the professional user perspective, presenting the Local Guide at IFA 2003 to content providers like broadcasters and journalists showed that there was a strong interest in such novel DVB-T based services. For broadcasters the technological innovations of the CONFLUENT project open up the chance to offer a free push service to its audience, later combined with personalised hybrid features. Moreover, the service shows one innovative solution of repurposing content deriving from content providers content resources such as TV, radio and the Internet. Also of interest to broadcasters is the fact that the MHP application can run on standard MHP set-top boxes as demonstrated on the Activy 300 at IFA 2003. This option enables viewers to access the Local Guide on their TV screens via the set-top box (STB) and navigate their way through the various menu points using the STB remote control. Using this option, viewers can plan a trip in advance from the comfort of their own home. 


\section{$4 \quad$ Application development}

The technical implementation of the Travel Guide application is based on concepts generated in the former MCP project. Basically the service Travel Guide is a classical MHP application (Xlet), developed at the TU-BS. That means it is executable in stable way on every MHP compliant terminal. MHP was developed originally for the classical home environment, interactive services in the living room. To make such a platform mobile it makes sense to consider some mobile use cases that require additional interfaces from the execution environment. So, based on the enhancements of the MHP API in the MCP project, the CONFLUENT project optimised and implemented the additional interfaces and programmed some services, the most important is the Travel Guide, to proof the concept. Technically this is possible because the application code, layout XML-files and content is strictly separated. For the structuring of content, an XML format has been defined, which also allows for the location referencing of the content. The rendering engine GUIMAN is rendering the layout files and is thus de-coupling the application from layout and user interaction.

The communication layer for interaction with the mobile phone via Bluetooth allows the MCP terminal to act as a server to allow a mobile phone the access to its multimedia content.

\section{$5 \quad$ Static main MHP-Automotive terminal}

Basically this terminal is a kind of mock-up car. The car display and the original VW Touareg steering wheel are integrated in a car dashboard mock-up, see Figure 3. This terminal is the Multimedia Car Platform terminal that was originally developed in the EU project Multimedia Car Platform (MCP) and is currently updated for full MHP 1.0.3 compliance [1].

Figure 3 Static main MHP-Automotive terminal 


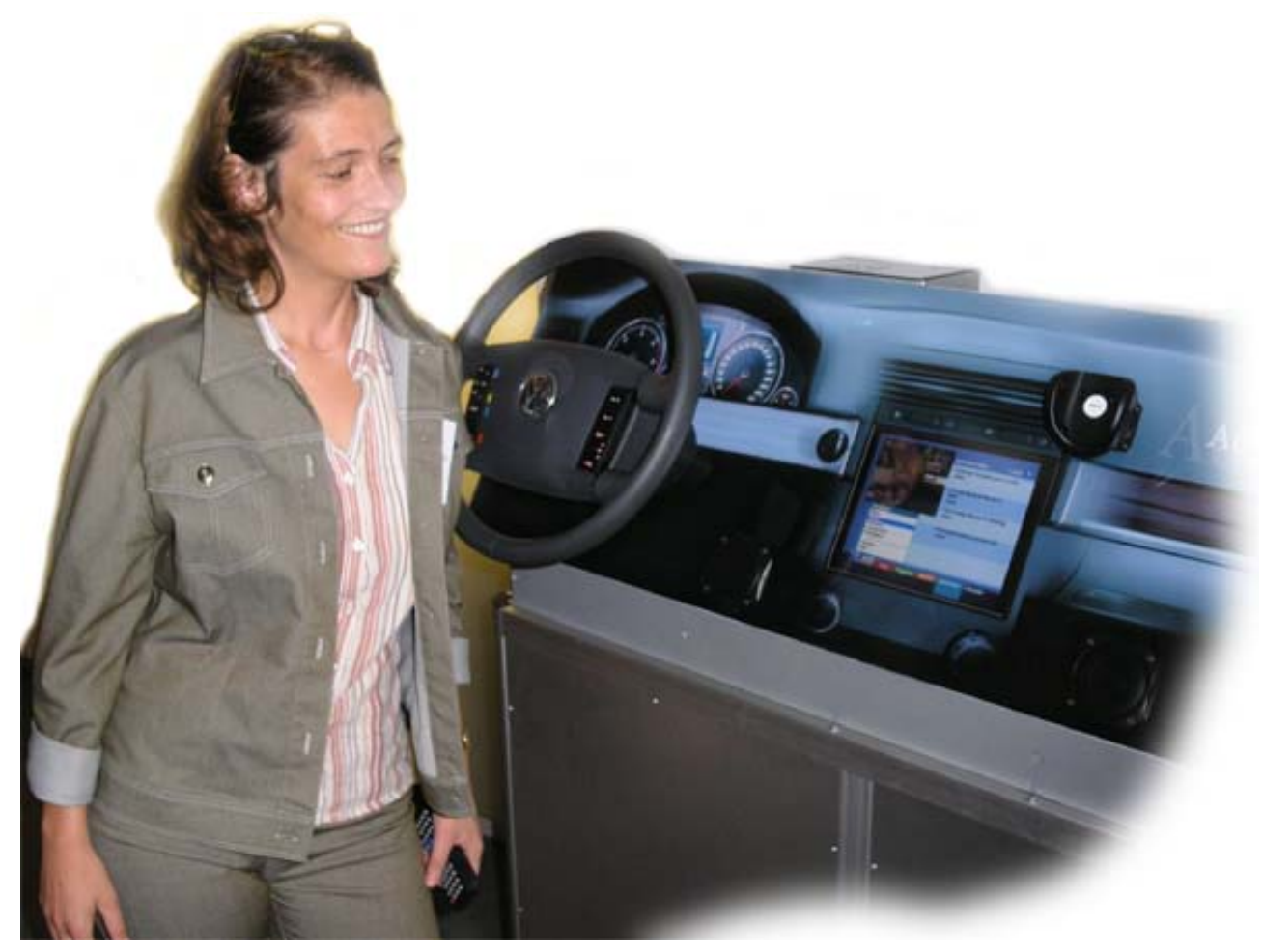

A Windows XP PC with all necessary hardware interfaces (e.g. DVB-T card, Bluetooth adapter, GPS-receiver, keyboard splitter, sound card for microphone array) and sufficient processing power to run the MHP Reference Implementation of IRT was installed in the mock-up dashboard.

On top of the Windows Operating System, a JAVA Virtual Machine with some extensions was installed. To enhance the functionality of the MHP-based applications a number of MCP specific API's for navigation and positioning, speech, Bluetooth link to mobile phone, communications, broadcast and MHP compliant application management were implemented by TU-BS. Further information on these API's is given in section 11.

The MCP terminal extracts the Travel Guide content directly from the object carousel transmitted via DVB-T. On the MCP platform calls are made to carousel for accessing additional media whereas on an IRT MHP compliant terminal (a laptop) with an MHP reference implementation software stack, described in section 9, calls may be made to files on a storage manager developed for the project as described in section 12.

The Travel Guide application when running on either the MCP terminal or the IRT terminal can take a subset of the content using a mechanism on the host for transfer to a third terminal, e.g. a mobile phone, using Bluetooth. Due to the fact that current mobile phones do not provide enough resources to provide an MHP stack, only the content is transmitted to the phone. In this specific case this means that for data exchange with the mobile phone, the Travel Guide offers a subset of its multimedia content via a Bluetooth connection.

A Symbian client application running on the mobile phone realises the service by extracting the subset of Travel Guide content on the mobile phone. 
NetiKOS demonstrated in co-operation with T-Systems, RBB, and TU-BS the transferability and scalability of content in DVB-T and 3G network environments in general, and between MHP-based terminals and S-60 Symbian OS mobile phones in particular. The demonstration was based on the Local Guide Potsdam where locationbased content is available through multiple channels.

Data transfer between the CONFLUENT terminal and the mobile phone is based on transferring content files using the OBEX protocol on Bluetooth, enabling a transfer range of up to $10-15 \mathrm{~m}$. After having been transmitted from the MHP-based terminal, the CONFLUENT application running in the background on the phone automatically detects the incoming content, and presents it to the user without any additional acceptance or intervention.

The CONFLUENT S-60 application was written in Symbian $\mathrm{C}++$ for maximum robustness and portability. The application, excluding the video clip player, is only 27 $\mathrm{kB}$ in size. Using Bluetooth, data transfer between the MHP-based terminal and the phone takes place in a matter of seconds.

When available (e.g., Nokia-3650), the application and associated content may be stored on the external memory card. Within the Local Guide Potsdam demonstrator, the content of a typical sight (5 images, 7 text extracts, total of $4 \mathrm{~min}$ of video clips) consumes approximately 1.3 MB of disk space.

\section{$7 \quad$ Activy set-top box}

The MHP-based Travel Guide application was demonstrated to run on a standard settop box: The Fujitsu-Siemens “ Activy 300". The Activy 300 is based on PC Hardware:

1 Microprocessor: Intel Celeron $733 \mathrm{MHz}$, Intel 810.

2 Storage: $256 \mathrm{MB}$.

3 Graphic: Twia 500 with TV out (PAL 520x 576, 50 Hz, 16:9), MPEG2 HW Decoder Sigma Design.

4 Audio: AC 97/ 16 bit stereo and S/PDIF (AC-3/MPEG2 Audio) Output, Audio standard support for DTS and Dolby Digital (5.1 / AC3).

5 120GB HD, DVD, LAN on board, embedded XP etc.

The Activy 300 allows for the running of an MHP Reference Implementation. For this demonstration the IRT MHP Reference Implementation (1.02) provided by RBB was used. In addition, MPEG 1/2 streams from DVD, VB and IP can be received, decoded via an MPEG decoder chip and accessed via TV. Locally stored MPEG4 video clips can be displayed via a software decoder/player. With sufficient bandwidth available in the DVB-T broadcast stream integration into the MPEG 2 transport stream via IP insertion and storage on hard disc are possible. As playout bandwidth at IFA 2003 was limited to $300 \mathrm{kbit} / \mathrm{s}$ the demonstration on the Activy was limited to the Travel Guide Application without the additional MPEG 4 videos. In an iterative testing and feedback process, the Travel Guide application, originally programmed for the 
automotive PC system was adapted to the Activy STB. Problems and inconsistencies (i.e. in font size, display etc.) were reported and appropriate amendments were incorporated into the next version until the application ran smoothly on both terminals.

At IFA2003 an Audi A6 was equipped with an embedded system (DVB-T tuner and embedded MHP implementation), see Figure 4. Several mobile services were demonstrated on this platform such as TV, news ticker, CONFLUENT Travel Guide, etc. This showcase was set up by the MHP-Automotive forum in close cooperation with the CONFLUENT project. Partners in the showcase were: T-Systems Nova, TUBS, Fuba/Delphi, DiBcom, ICT Embedded B.V. and Philips.

Figure 4 MHP automotive terminal MHP

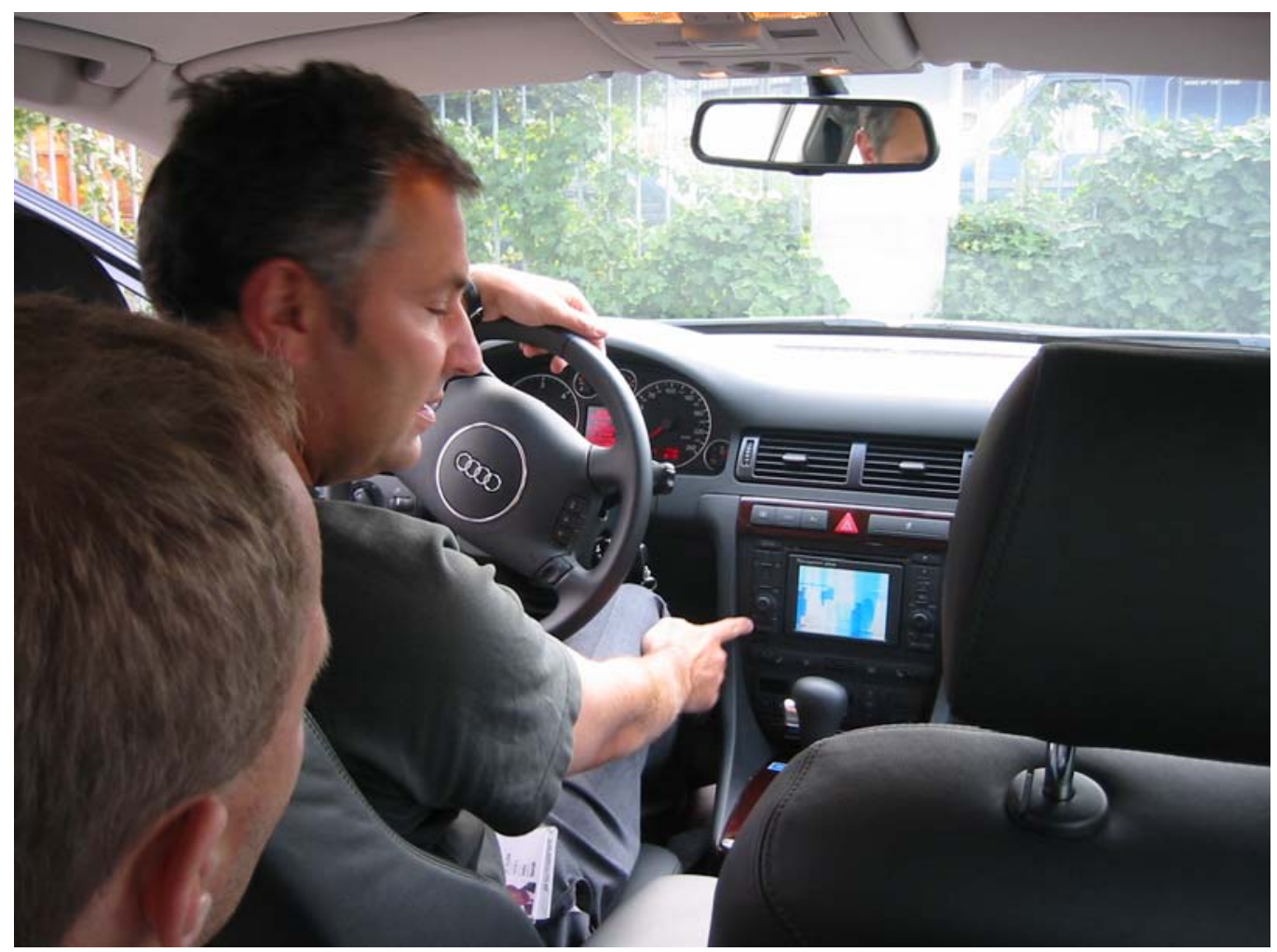

As MHP is a platform supporting a worldwide horizontal market for the delivery of services, it was an interesting idea to use the same platform in a car environment. Extending the platform with automotive related API's like speech, positioning, etc. the platform enables interesting new (location-based) services. Furthermore, being close to the mass consumer market, all ingredients like hardware, bandwidth and a huge variety of services are available at low-cost.

Starting from these considerations, the EU project Multimedia Car Platform was initiated. It provided a first showcase at IFA 2001 and a draft specification for the MHP extensions. In 2002, car manufacturers, suppliers and broadcasters interested in promoting the idea of an MHP Automotive established the MHP Automotive (MCP) 
forum. At the same time, the DVB project initiated a working group for the commercial requirements of MHP automotive.

ICT Embedded B.V. developed the MHP embedded software for IRT. The resulting embedded MHP middleware was optimised for set-top boxes and integrated receiver devices where small footprint, high performance, and highest robustness are essential requirements. Using the ISO 9126 architectural model supporting Quality Attributes for non-functionality requirements (like portability, error-handling, maintainability, footprint, etc.) the complete MHP software stack was enhanced.

This MHP Reference Implementation was implemented on a Philips PC board (NEXPERIA). Delphi / Fuba provided the DVB-T front end for the showcase. At IFA 2003 an Audi A6 was equipped with the Fuba DVB-T front-end and the ICT Embedded B.V. / Philips MHP implementation (an extra box with a car PC and embedded MHP implementation). The car integration was organised by T-Systems.

The Blaupunkt entertainment unit, including the liquid crystal display could be reused as well as the FUBA diversity antenna system of the A6. Fuba's digital DVB$T$ receiver replaced the analogue TV receiver of Fuba. This DVB-T receiver included the Dibcom chipset for diversity reception that was developed in the CONFLUENT project. The quality of the digital TV signal was excellent with zero visible errors in the surroundings of the fair. Mobile reception of DVB-T in the car has become a reality due to the strong support of European projects (MOTIVATE, MCP and CONFLUENT) and is no longer a research issue.

\section{The terminal of IRT}

The demonstrations of the CONFLUENT project at the IRT booth at IFA 2003 in Berlin were aiming at:

1 Demonstration of the CONFLUENT project content on the IRT terminal.

2 Showing the concepts of the CONFLUENT project on the network side to enable the encoding of the significant common application "Travel Guide Potsdam"

To demonstrate the concept of the convergence of broadcast and mobile telecommunication with MHP as a basis IRT demonstrated at IFA 2003 different laptop-based applications (one laptop was a kind of tablet PC where you could "hide" the keyboard by turning the screen; the second was an ordinary laptop computer). All applications were running on IRT`s MHP Reference Implementation Version 103R1_1 with MPEG-4 QuickTime Plug-In.

The "Travel Guide Application" was received inside the exhibition hall and decoded by the IRT terminals. This demonstrated the compatibility of the CONFLUENT project application with the IRT terminals thanks to MHP as the enabling platform

One important feature of the CONFLUENT Travel Guide application is MPEG-4 streaming or file transfer over the broadcast channel in addition to the common MPEG-2 audio/video. By means of an MHP application the principle of transmitting MPEG-4 additional content was demonstrated at IFA 2003. Over WLAN, the terminal requested a list of additional video clips available on the Broadcaster's WebServer. The user can then choose one of the videos (here: attractions of Potsdam). 
Subsequently, the video is sent to the terminal via the broadcast channel, using multi-protocol encapsulation (MPE).

To demonstrate the concept of using a back-channel (WLAN/GPRS) for various purposes, IRT has also developed an E-Mail application based on MHP. The Reference Implementation gives the opportunity of supporting different kinds of back-channels (Modem, ISDN, DSL, (W)LAN) for interactive TV programs and applications. At IFA 2003 visitors had the possibility to send emails directly from the MHP terminal.

\section{Mobile terminal in IRT car}

The aim of the final In-Car demonstration of CONFLUENT at IFA 2003 in Berlin was:

1 To compare different receiver types under mobile reception conditions and to proof the superiority of the diversity receiver concept.

2 To combine the achievements of mobile DVB-T reception with a MHP receiver inside the demonstration car.

The essential thing about this demonstration was the combination of the enhanced DVB-T receiver technology and the MHP based terminal showing the CONFLUENT application "Travel Guide Potsdam" in mobile reception.

\section{Extensions of the MHP}

The Multimedia Home Platform (MHP) is originally a software platform to present services and interactive applications of various providers on digital television receivers and set-top boxes. These include e-commerce services, interactive game shows, traffic and weather information, news and stock market reports.

The applications and software modules demonstrated in CONFLUENT are compliant to the MHP 1.0.3 specification. In addition to the original specification the software developed in CONFLUENT includes extensions for positioning, via an interface to the navigation system, Bluetooth, speech control and MPEG 4.

MHP speech API

Various mobile multimedia devices nowadays are already using speech input as additional user interface. To enable this functionality also to broadcast multimedia applications a new small MHP Speech API was defined and implemented. When an application arrives on a mobile platform it starts to detect whether a speech engine (including the API implementation) is available. If this is the case, the user can control the application via voice in addition to other input methodologies. This includes also speech output for introduction texts and context sensitive help functions.

If there is no speech function installed on the terminal, the application can be used with the existing input devices. For example, with a remote control on a TV set.

MHP Navigation API

Location based services are one of the most attractive new service types on mobile devices like cars, PDAs and mobile phones. They help the mobile users to find shops, restaurants, museums, friends or their children. They allow for location-based 
advertisement, guidance through public places or immediate support in case of emergency. To support location-based services in an MHP environment, an API was developed which allows applications to:

1 Access the position of the end-user devices, independent of the underlying positioning system

2 Start and control a third party navigation system if available

3 Display a scalable Navigation map, embedded in the MHP-application

4 Allow for high security of position (access rights to position information can be controlled by the platform and the user) with full support of MHP security architecture.

An implementation of the API was presented that is used by the Travel Guide for a tourist guide scenario.

A Java-based Navigation application was embedded into the MHP. Teleatlas provided the map database for Potsdam.

MHP Bluetooth API

In the last few years a new short-range communication standard has become very common. Bluetooth easily connects additional devices such as handhelds, PDAs, etc. Various terminals, first of all mobile phones, nowadays have such a radio interface. To be able to transfer content from a Java based platform via Bluetooth to another terminal it was necessary to integrate an appropriate API.

By pressing a button within one sight of the Travel Guide all the content related to this sight is transferred to the mobile phone using the Bluetooth OBEX protocol. The Travel Guide "pushes" a selected part of the content into the Inbox of a mobile phone. There it is picked up by the pre-installed client application.

\section{MHP MPEG-4}

The Travel Guide presents a rich audio-visual set of information on Potsdams sights. The most eye-catching information is the MPEG-4 videos on the MHP based platform as well as on the mobile phone. This functionality was enabled by an MPEG-4 plug-in for the MHP.

The videos have been integrated seamlessly into the Travel Guide application and start automatically if information on a sight is requested.

MHP application navigator

In addition, a new MHP application navigator was developed that allows for the control of multiple simultaneously running MHP applications and for access to digital terrestrial television. The navigator was especially developed for a car environment and is designed to allow easy integration into existing car multimedia systems. Its security architecture fully controls the lifecycle of applications and so can for example stop an application (which is not designed for driving assistance) if the car is starting to drive faster than for example $3 \mathrm{~km} / \mathrm{h}$.

The navigator is fully controllable via voice commands like all the other applications.

\section{The storage manager}


The Travel Guide application was integrated by Brunel University on an IRT terminal (laptop) so that it can be accessed through a selection on its user interface and was developed as a separate application with the tools that were developed through a number of EU projects. The process of developing convergent multimedia services for delivery over DVB and UMTS networks for the CONFLUENT project system consisted of four main activities: Defining and fast prototyping the service scenario for early user trials [2]; Provisioning the Service with Content and Indexing data [3]; Configuring a service provisioning system [4]; Accessing the Service from a layered terminal architecture for consuming the service [5].

The application level architecture of the laptop consists of a User Interface Rendering System (UIRS), a Control Manager (CM), a Storage Manager (SM), an Electronic Service Guide (ESG), a Network Manager (NM) and an Event Manager (EM) [5]. The service scenario of the CONFLUENT project called for the incorporation of a Bluetooth interface to a Nokia 7650 mobile phone to access a subset of the media from the laptop. Thus the architecture was enhanced with a Bluetooth interface that allows media to be accessed from mobile phones.

On the laptop the Travel Guide application is bound in with location based services. These include a location service and a message service providing MPEG-4 audio/video clips, pictures and texts associated with a physical attraction or an event at a location respectively. For all locations each attraction is indexed using MPEG-7 and associated with related media for both DVB-T and GPRS transmission using Internet Protocol.

\section{Conclusions}

The availability of standardized API's adapted to a hybrid UMTS and DVB-T network and to the technical limitations of mobile/portable terminals is crucial to the commercial exploitation of any service combining UMTS and DVB-T. In this paper work carried out in the European Commission Framework Program 5 (FP5) project CONFLUENT demonstrating the feasibility of such API's by enabling the reception of a Multimedia Home Platform (MHP) based application transmitted over DVB-T on five different terminals with parts of the service running on a mobile phone has been reported.

A set of common functionalities has been extracted from the enhanced API profiles and published as a proposed minimum set of functionalities for a future MHP-MOBILE approach intended to be the standard for API development in conformant hand-held/portable/mobile terminals.

In the CONFLUENT project a user interaction means with the broadcast part was developed where the broadcast receiver is detached from an end-user interaction device, a Bluetooth capable mobile phone, and a client-server application between both terminals was defined. This paves the way for the concept of "pervasive broadcast receivers" where numerous (low-cost) broadcast receivers with specific features like display, user interaction means, multimedia, capabilities and storage can be massively deployed in public places. New applications could then be developed building on the end-user terminal mobile capabilities to communicate peer-to-peer thanks to, for example, Bluetooth links. In this way end-user terminal complexity can be limited while still providing broadcast-based applications. New applications building on these features could come from the e-government/e-citizen domains. 
It is important to note that while this paper contributes to establishing the technical feasibility of services combining UMTS and DVB-T crucial to the success of such services will be the creation of new business models [6]. The European Commission Framework Program 6 (FP6) Integrated Project INSTINCT has strong focus on developing such business models [7]. The specification of the INSTINCT project was a key output of the CONFLUENT project. The critical role of consumer behaviour research ([8]) in establishing viable business models is addressed in the INSTINCT project through the identification of user acceptability factors. The broader challenges facing mobile services in general are discussed in [9].

\section{Acknowledgements}

The authors gratefully acknowledge the support for this work that is funded by the EU under the IST program in the project CONFLUENT (Enabling commercial application of convergent services in collaborative networks of UMTS and DVB-T systems), IST-2001-38402. www.brunel.ac.uk/projects/confluent

\section{References}

1 Klinkenberg, F. and Schiek, U. (2003) 'Extension of the Multimedia Home Platform for Applications in an Automotive Environment', IEEE International Conference on Consumer Electronics, Los Angeles, US

2 Cosmas, J., Itagaki, T., Cruickshank, L., Elgohari, L., Harris, G. and Lucas, A. (2003)

'Simulation and User Trial Results of a Converged DVB-T and GPRS Sports Service', Proceedings $4^{\text {th }}$ Conference. on Telecommunications, Aviero, Portugal, pp 411-414.

3 Berg, M., Cosmas, J., Itagaki, T., Cruickshank, L., Lucas, A., Krishnapillai, K., Heng, L. and Elgohari, L. (2003) 'Converged DVB-T and GPRS Service Scenarios and Application Production Tools', Proceedings $4^{\text {th }}$ Conference on Telecommunications, Aviero, Portugal, pp 471-474.

4 Buttterfield, S., Garrec, D., Cosmas, J., Itagaki, T., Lucas, A., Krishnapillai, K., Heng, L., Haque, M., Sun, F. and San Martin, M. (2003) 'CISMUNDUS: Terminal Hardware, Middleware and Applications', Proceedings $4^{\text {th }}$ Conference on Telecommunications, Aviero, Portugal, pp 269-272.

5 Benali, O., Casagranda, P., Cosmas, J., Garrec, D., Guiraudou, M., Itagaki, T., Kridhnapillai, K., Lefevre, F., Lucas, A., Martinez, G., Mazieres, B., Milanesio, D. and Heng, L., (2003), 'Architecture and Proof-of-Concept Implementation for Mobile/Broadcast Hybrid Networks', Proceedings $4^{\text {th }}$ Conference on Telecommunications, Aviero, Portugal, pp 59-62.

6 Sigurdson, J. and Ericsson, P., (2003), 'New services in 3G - new business models for streaming and video', International Journal of Mobile Communications, Vol. 1, Nos 1/2, pp 15-34.

7 http://www.ist-instinct.org

8 Vrechopoulos, A., Costantiou, I., Sideris, I., Doukidis, G. and Mylonopoulos, N., (2003), 'The critical role of consumer behaviour research in mobile commerce', International 
Journal of Mobile Communications, Vol. 1, No 3, pp 329-340.

9 Keng Siau and Zixing Shen, (2003), 'Mobile communications and mobile services', International Journal of Mobile Communications, Vol. 1, Nos 1/2, pp 3-14. 